\section{Wine: looking ahead}

\section{Matteo Bordiga}

Dipartimento di Scienze del Farmaco, Università del Piemonte Orientale A. Avogadro, Novara, Italy

\section{In vino veritas}

Pliny the Elder

It is with great pleasure that I have been asked to follow Professor Maurizio Trevisan as the new Editor-in-Chief of Wine Studies. As new Editor-in-Chief, I will try to bring new aspects to the publication and will share these ideas. I have ambitious plans for the Journal in order to improve its strengths as the international outlook, the emphasis on manuscripts which translate theoretical research into practical and applied technologies for the wine industry, and above all the high regard the Journal holds in both industry and Academia.

Several studies have cited the Mediterranean-style diet as an example of health-promoting eating. In fact, the Mediterranean diet has become the reference diet for the prevention of cardiovascular and metabolic diseases. The diet itself represents a concept that varies across the Mediterranean region but is characterized by relatively high intakes of olive oil, legumes, fruits and vegetables, unrefined cereals, fish, and red wine. Red wine seems to be an essential component of the diet, since moderate consumption of wine is associated with lower risk and mortality from cardiovascular disease. Oxidative stress associated with atherosclerosis and endothelium dependent vascular inflammation plays a major role in the development of cardiovascular disease. Red wine contains bioactive components like proanthocyanidin, quercetin, resveratrol and these active compounds exert protective functions like free radical scavenging effects, decreasing the oxidative stress and reducing the inflammatory atherosclerotic lesion. The most active polyphenols present in red wine are flavonoids, important due to their putative antioxidant properties (e.g. the
French Paradox phenomenon).

Moreover, if we consider that the history of civilization is, in many ways, the history of wine we perfectly understand its past, present and future impact on our society. Man has been drinking wine for the last 9000 years and the medical profession has been using it as a medicine for the past 5000 years, making it the oldest medicine. Hence, it is crucial to continue studying new effects of well-known compounds and, at the same time, searching novel bioactive molecules. The wine industry needs to change people's perception of wine from a beverage drunk occasionally to one drunk daily (in moderation) for pleasure and health benefits, able to enjoy life physically, mentally, and environmentally.

However, while the importance of wine as final product is undeniable, it is also significant to focus our attention on the entire production process: from vine to glass. The evolving scientific and technological practices, that are now influencing how grapes are grown and how wine is made, characterize a very complex landscape but rich in hints (e.g. wine chain by-products valorization).

Vine cultivation and wine making process generate a significant number of wastes and by-products. These materials include pruning, stem, pomace and seeds, yeast lees, tartrate, and wastewater. Generally, only a very small portion of these materials is used (e.g. fertilizer, animal feed, or fuel production). Over the past few years, wine making by-products have attracted considerable attention as potential sources of bioactive phytochemicals, which could be used for various purposes in the pharmaceutical, cosmetic and food industry. Considering the challenges in the area of food industry, efforts need to be made to optimize food processing technology and minimize the amounts of by-product waste. Food industry is generating increasing amounts of by-products all along the chain of food production. The most important means of waste minimization is the application of more efficient production technologies. Still, by-product generation is inevitable. Environmental regulations and high waste discharge costs have forced food
Correspondence: Matteo Bordiga, Dipartimento di Scienze del Farmaco, Università del Piemonte Orientale A. Avogadro, Largo Donegani 2, 28100 Novara, Italy.

Tel. +39.0321.375602 - Fax: +39.0321 .375621 .

E-mail: matteo.bordiga@pharm.unipmn.it

Key words: Wine Sudies, Editor-in-Chief, PAGEPress.

This work is licensed under a Creative Commons Attribution 3.0 License (by-nc 3.0).

(C) Copyright M. Bordiga, 2014

Licensee PAGEPress, Italy

Wine Studies 2014; 3:2186

doi:10.4081/ws.2014.2186

processors to find ways to better treat and utilize processing wastes. Efficient utilization of food processing by-products is important for the profitability of the food industry. By-products and wastes of plant-based food processing, which represent a major disposal problem for the industry concerned, are very promising sources of value added substances, with particular emphasis being given to the retrieval of bioactive phytochemicals and technologically important secondary metabolites. Over the next few years, the area of food processing waste management will expand rapidly. Future legislation regarding industrial waste - including that of wineries - will become even more demanding, thus increasing the cost of waste management. The recovery of added value products can help in that direction. These recovery processes are part of a new philosophy of sustainable agriculture.

All this considered, we can conclude that Wine Studies is now intended to be a forum to investigate and discuss new and significant researches related to the wine supply chain aiming to promote these arguments worldwide. We hope Readers will find the journal interesting and will actively contribute to its development. 